\title{
On Stress-Strength Interval-System Reliability with Applications in Heart Conditions
}

\author{
Hoang Pham \\ Department of Industrial and Systems Engineering, \\ Rutgers University, New Jersey, 08854, USA. \\ E-mail: hopham@ soe.rutgers.edu
}

(Received August 12, 2019; Accepted September 21, 2019)

\begin{abstract}
The random variable $X$ represents the stress placed on the system by the operating environment and random variable $Y$ represents the strength of the system. A system is able to perform its intended function if its strength is greater than the stress imposed upon it. Reliability of the system is defined as the probability that the system is strong enough to overcome the stress. That is, $R=P(Y>X)$. In other words, reliability is the probability that the strengths of the unit are greater than the stresses. The stress-strength model has found interests in many applications include mechanical engineering and human heart monitoring conditions. The interval-system is defined as a system with a series of chance events that occur in a given interval of time. A $k$-out-of- $n$ interval-system is a system with a series of $n$ events in a given interval of time which successes (or functions) if and only if at least $k$ of the events succeed (function). In short, the $k$-out-of- $n$ interval-system is an interval-system which successes if and only if at least $k$ of $n$ events succeeds. The stress-strength reliability inference of the interval-system with a series of $n$ independent events that occurs in a given interval of time is considered. The reliability of the interval-system is the probability that at least $k$ out of $n$ events in a given interval of time succeed. This paper derives uniform minimum variance unbiased and maximum likelihood reliability estimates of $k$-out-of- $n$ interval-system based on stress-strength inference events where $X$ (stress) and $Y$ (strength) are independent two-parameter exponential random variables. An application in human heart conditions to illustrate the results is discussed.
\end{abstract}

Keywords- $k$-out-of- $n$ system, System reliability, Heart monitoring condition, UMVUE, Stress strength interval system.

\section{Introduction}

There has been continuous interest in the problem of estimating the probability that one random variable exceeds another, that is, $R=P(Y>X)$ where $X$ and $Y$ are independent random variables. This problem arises in the classical stress-strength reliability where one is interested in assessing the proportion of the times the random strength $Y$ of a component exceeds the random stress $X$ to which the component is subjected (Kotz et al., 2003). In other words, stress-strength reliability model is defined as the life of a component being exposed to $X$ stress and having $Y$ strength. This implies that if stress exceeds strength $(X>Y)$ then it will be impossible for a component to survive. The reliability of such a component which consists of stress and strength is expressed as $R=P(Y>X)$.

The stress-strength reliability model for various distributions of $X$ and $Y$ random variables has been studied in past years (Bhattacharyya and Johnson, 1974; Beg, 1980; Chao, 1982; Weerahandi and Johnson, 1992; Kunchur and Munoli, 1993; Aminzadeh, 1997; Hanagal, 2003; Kotz et al., 2003; Guo and Krishnamoorthy, 2004; Kundu and Gupta, 2005; Saracoglu and Kaya, 2007). Some practical examples including gear transmission systems can be found in Weerahandi and Johnson (1992) and Wei et al. (2019). For example, Weerahandi and Johnson (1992) presented a rocket-motor experiment data where $Y$ represents the chamber burst strength and $X$ 
International Journal of Mathematical, Engineering and Management Sciences

Vol. 5, No. 1, 1-12, 2020

https://doi.org/10.33889/IJMEMS.2020.5.1.001

represents the operating pressure assuming that $X$ and $Y$ are independent normal random variables. Saraçoglu and Kaya (2007) studied stress-strength reliability problems for Gombertz distribution, Beg (1980) for the two-parameter exponential distribution, Kundu and Gupta (2005) for a generalized exponential distribution. Kunchur and Munoli (1993) discussed the reliability estimation for a multi-component stress-strength model based on exponential distributions.

On the reliability estimation, there are numerous studies (Laurent, 1963; Rutemiller, 1966; Lehmann and Casella, 1998; Pham, 2010, 2018) on the reliability estimation of systems where components are connected either in series or parallel based on the method of maximum likelihood estimate (MLE) or uniform minimum variance unbiased estimated (UMVUE). Many researchers assumed that systems comprised of units that follow exponential failure distribution function (Laurent, 1963; Rutemiller, 1966). Some researchers recently discussed UMVUE and MLE of reliability for $k$ out of $n$ systems which are composed of independent and identically distributed components with two-parameter exponential lifetimes for both the uncensored and censored failure cases (Lehmann and Casella, 1998; Kotz et al., 2003; Pham, 2010; Pham and Pham, 2010; You and Pham, 2016).

In this study, we introduce an interval-system, which is defined as a system with a series of chance events that occur in a given interval of time. The $k$-out-of- $n$ interval-system is also defined as a system with a series of $n$ events in a given interval of time which successes (or functions) if and only if at least $k$ of the events success (function).

In this paper we discuss the reliability estimates of $k$-out-of- $n$ interval-system based on stressstrength events where $X$ (stress) and $Y$ (strength) are independent two-parameter exponential random variables using the UMVUE and MLE methods. A numerical application in human heart conditions is discussed to illustrate the model results.

\section{Estimation of Stress-Strength Reliability Function}

In this section we discuss statistical inferences on the stress-strength reliability event based on the method of uniform minimum variance unbiased estimate and maximum likelihood estimate.

Let $X$ be the random variable that represents the stress placed on the system by the operating environment and $Y$ represents the strength of the system. A system is able to perform its intended function if its strength is greater than the stress imposed upon it. Reliability of the system is defined as the probability that the system is strong enough to overcome the stress, that is $R=P(Y$ $>X$ ), where $X$ and $Y$ are independent random variables with the following two-parameter exponential pdf.

$$
f_{X}(x)=\frac{1}{\sigma_{x}} e^{-\frac{\left(x-\mu_{x}\right)}{\sigma_{x}}} \quad \text { for } x>\mu_{x}, \mu_{x} \geq 0, \sigma_{x}>0
$$

and

$$
f_{Y}(y)=\frac{1}{\sigma_{y}} e^{-\frac{\left(y-\mu_{y}\right)}{\sigma_{y}}} \quad \text { for } y>\mu_{y}, \mu_{y} \geq 0, \sigma_{y}>0
$$

respectively. 
International Journal of Mathematical, Engineering and Management Sciences

Vol. 5, No. 1, 1-12, 2020

https://doi.org/10.33889/IJMEMS.2020.5.1.001

\subsection{UMVUE and MLE of Reliability Function}

In this paper, we assume that $\sigma_{x}$ and $\sigma_{y}$ are known. We plan to report the modeling results when both $\sigma_{x}$ and $\sigma_{y}$ are unknown in a near future the stress-strength reliability can be obtained as follows.

\section{Reliability Function}

When $\mu_{y} \geq \mu_{x}$ : The stress-strength $R$ is given by

$$
\begin{aligned}
P(Y>X) & =P_{X} P_{Y \mid X}\left(Y>X \mid \mu_{x}<X<\mu_{y}\right)+P_{X} P_{Y \mid X}\left(Y>X \mid X>\mu_{y}\right) \\
& =\frac{1}{\sigma_{x}} \int_{\mu_{x}}^{\mu_{y}} e^{-\left(\frac{x-\mu_{x}}{\sigma_{x}}\right)} d x+\frac{1}{\sigma_{y}} \int_{\mu_{y}}^{\infty} e^{-\left(\frac{x-\mu_{y}}{\sigma_{y}}\right)} e^{-\left(\frac{x-\mu_{x}}{\sigma_{x}}\right)} d x \\
& =1-\frac{\sigma_{x} e^{\left(\frac{\mu_{x}-\mu_{y}}{\sigma_{x}}\right)}}{\sigma_{x}+\sigma_{y}} .
\end{aligned}
$$

When $\mu_{y} \leq \mu_{x}$ : The stress-strength $R$ is given by

$$
\begin{aligned}
P(Y>X) & =E_{X} P_{Y \mid X}(Y>X \mid X) \\
& =\frac{1}{\sigma_{x}} \int_{\mu_{y}}^{\infty} e^{-\left(\frac{x-\mu_{y}}{\sigma_{y}}\right)} e^{-\left(\frac{x-\mu_{x}}{\sigma_{x}}\right)} d x \\
& =\frac{\sigma_{y} e^{\left(\frac{\mu_{y}-\mu_{x}}{\sigma_{y}}\right)}}{\sigma_{x}+\sigma_{y}} .
\end{aligned}
$$

From Eq. (3) and (4), the stress-strength reliability function $R$ can be written as,

$$
R=P(Y>X)=\left\{\begin{array}{cc}
1-\frac{\sigma_{x} e^{\left(\frac{\mu_{x}-\mu_{y}}{\sigma_{x}}\right)}}{\sigma_{x}+\sigma_{y}} & \text { for } \mu_{y} \geq \mu_{x} \\
\frac{\sigma_{y} e^{\left(\frac{\mu_{y}-\mu_{x}}{\sigma_{y}}\right)}}{\sigma_{x}+\sigma_{y}} & \text { for } \mu_{y} \leq \mu_{x}
\end{array}\right.
$$

Assume independent random samples $X_{1}, X_{2}, \ldots, X_{m}$ and $Y_{1}, Y_{2}, \ldots, Y_{a}$ are drawn from the stress pdf $f_{X}(x)$ and strength pdf $f_{Y}(y)$ given in Eq. (1) and Eq. (2), respectively. Let $X_{(1)}, X_{(2)}, \ldots, X_{(m)}$ and $Y_{(1)}$, $Y_{(2)}, \ldots, Y_{(\mathrm{a})}$ be the corresponding ordered statistics. It can be shown that $X_{(1)}$ and $Y_{(1)}$ are complete sufficient statistic for $\mu_{x}$ and $\mu_{y}$, respectively, when $\sigma_{x}$ and $\sigma_{y}$ are known.

\section{UMVUE of $\boldsymbol{R}^{k}$}

We now wish to obtain the statistical function $T_{k}$, the UMVUE of $R^{k}=(P(Y>X))^{k}$ where $\sigma_{x}$ and $\sigma_{y}$ are known. From Eq. (5), we have: 
International Journal of Mathematical, Engineering and Management Sciences

Vol. 5, No. 1, 1-12, 2020

https://doi.org/10.33889/IJMEMS.2020.5.1.001

When $\mu_{y} \geq \mu_{x}$

$$
\begin{aligned}
R^{k} \equiv(P(Y>X))^{k} & =\left(1-\frac{\sigma_{x}}{\sigma_{x}+\sigma_{y}} e^{-\left(\frac{\mu_{y}-\mu_{x}}{\sigma_{x}}\right)}\right)^{k} \\
& =\sum_{i=0}^{k}(-1)^{i}\left(\begin{array}{l}
k \\
i
\end{array}\right)\left(\frac{\sigma_{x}}{\sigma_{x}+\sigma_{y}}\right)^{i} e^{-\frac{\left(\mu_{y}-\mu_{x}\right) i}{\sigma_{x}}} .
\end{aligned}
$$

When $\mu_{y} \leq \mu_{x}$

$$
\begin{aligned}
R^{k} \equiv(P(Y>X))^{k} & =\left(\frac{\sigma_{y}}{\sigma_{x}+\sigma_{y}} e^{\left.\left(\frac{\mu_{y}-\mu_{x}}{\sigma_{y}}\right)\right)^{k}}\right. \\
& =\frac{\sigma_{y}^{k}}{\left(\sigma_{x}+\sigma_{y}\right)^{k}} e^{\frac{\left(\mu_{y}-\mu_{x}\right) k}{\sigma_{y}}} .
\end{aligned}
$$

Thus, $R^{k}$ can be written as

$$
R^{k}= \begin{cases}\sum_{i=0}^{k}(-1)^{i}\left(\begin{array}{l}
k \\
i
\end{array}\right)\left(\frac{\sigma_{x}}{\sigma_{x}+\sigma_{y}}\right)^{i} e^{-\frac{\left(\mu_{y}-\mu_{x}\right) i}{\sigma_{x}}} & \text { for } \mu_{y} \geq \mu_{x} \\
\frac{\sigma_{y}^{k}}{\left(\sigma_{x}+\sigma_{y}\right)^{k}} e^{\frac{\left(\mu_{y}-\mu_{x}\right) k}{\sigma_{y}}} & \text { for } \mu_{y} \leq \mu_{x}\end{cases}
$$

Let $W=Y_{(1)}-X_{(1)}$. An unbiased estimator of $R^{k}$ is given by

$$
Z_{k}=\left\{\begin{array}{ll}
1 & \text { if } \mathrm{X}_{i} \geq Y_{i} \\
0 & \text { otherwise }
\end{array} \quad \text { for } i=1,2, \ldots, k\right.
$$

where $k<\min \{m, a\}$.

In general, the method of finding the UMVUE is to search for any unbiased statistic $T\left(X_{1}\right.$, $\left.X_{2}, \ldots, X_{\mathrm{k}}\right)$ and a complete sufficient statistic $\hat{\theta}$ if one exists. Then the UMVUE is given by $E\left[T\left(X_{1}, X_{2}, \ldots, X_{k}\right) \mid \hat{\theta}\right]$. From Lehmann-Scheffe theorem (Lehmann and Casella, 1998). The UMVUE of $R^{k}$ is

$$
\begin{aligned}
& E\left(Z_{k} \mid X_{(1)}, Y_{(1)}\right)=P\left(X_{1}<Y_{1}, X_{2}<Y_{2}, \ldots, X_{k}<Y_{k} \mid X_{(1)}, Y_{(1)}\right) \\
& \quad=P\left(X_{1}-X_{(1)}<Y_{1}-Y_{(1)}+W, X_{2}-X_{(1)}<Y_{2}-Y_{(1)}+W, X_{k}-X_{(1)}<Y_{k}-Y_{(1)}+W<Y_{k} \mid W\right)
\end{aligned}
$$


International Journal of Mathematical, Engineering and Management Sciences

Vol. 5, No. 1, 1-12, 2020

https://doi.org/10.33889/IJMEMS.2020.5.1.001

which will be a function of $W, \sigma_{\mathrm{x}}$ and $\sigma_{\mathrm{y}}$ since the distribution of $X_{i}-X_{(1)}$ and $Y_{\mathrm{i}}-Y_{(1)}$ for $i=1,2$, $\ldots, k$ do not involve $\mu_{x}$ and $\mu_{y}$, respectively.

Therefore, based on Lehmann-Scheffe theorem, an unbiased estimator of $R^{k}$ based on $W$ is the UMVUE of $R^{k}$. Note that, the pdf of $W$ where $W=Y_{(1)}-X_{(1)}$ is given by

$$
f_{W}(w)= \begin{cases}\frac{m a}{\left(a \sigma_{x}+m \sigma_{y}\right)} e^{-\frac{\left(\mu_{y}-\mu_{x}-w\right)}{\sigma_{x}}} & \text { for } \mathrm{w}<\left(\mu_{y}-\mu_{x}\right) \\ \frac{m a}{\left(a \sigma_{x}+m \sigma_{y}\right)} e^{-\frac{a\left(w-\left(\mu_{y}-\mu_{x}\right)\right)}{\sigma_{y}}} & \text { for } \mathrm{w} \geq\left(\mu_{y}-\mu_{x}\right) .\end{cases}
$$

Theorem 1: The statistic $T_{k}$ is the UMVUE of $R^{k}$ where $\sigma_{x}$ and $\sigma_{y}$ are known, $k<\min \{m, a\}$, and

$$
T_{k}=\left\{\begin{array}{l}
\frac{(a-k)\left(k \sigma_{x}+m \sigma_{y}\right) \sigma_{y}^{k-1}}{m a\left(\sigma_{x}+\sigma_{y}\right)^{k}} e^{\frac{\left(Y_{(1)}-X_{(1)}\right) k}{\sigma_{y}}} \mathrm{I}\left(Y_{(1)}<X_{(1)}\right) \\
\quad+\sum_{i=0}^{k}(-1)^{i}\left(\begin{array}{l}
k \\
i
\end{array}\right) \frac{(m-i)\left(a \sigma_{x}+i \sigma_{y}\right) \sigma_{x}^{i-1}}{m a\left(\sigma_{x}+\sigma_{y}\right)^{i}} e^{-\frac{i\left(Y_{(1)}-X_{(1)}\right)}{\sigma_{x}}} \mathrm{I}\left(Y_{(1)} \geq X_{(1)}\right) .
\end{array}\right.
$$

The Proof of Theorem 1 is in the Appendix.

\section{MLE of $\boldsymbol{R}^{k}$}

It can be shown that the maximum likelihood estimate of $\mu_{x}$ and $\mu_{y}$ where $\sigma_{x}$ and $\sigma_{y}$ are known from the pdf $f_{X}(x)$ and $f_{Y}(y)$, respectively, are

$\hat{\mu}_{x}=X_{(1)} \quad$ and $\quad \hat{\mu}_{y}=Y_{(1)}$

From Eq. (5), we have

$$
R^{j}(t)=\frac{\sigma_{y}^{j}}{\left(\sigma_{x}+\sigma_{y}\right)^{j}} e^{\frac{\left(\mu_{y}-\mu_{x}\right) j}{\sigma_{y}}} \mathrm{I}\left(\mu_{y} \leq \mu_{x}\right)+\sum_{i=0}^{j}(-1)^{i}\left(\begin{array}{l}
j \\
i
\end{array}\right)\left(\frac{\sigma_{x}}{\sigma_{x}+\sigma_{y}}\right)^{i} e^{-\frac{\left(\mu_{y}-\mu_{x}\right) i}{\sigma_{x}}} \mathrm{I}\left(\mu_{y} \geq \mu_{x}\right) .
$$

Thus, the MLE of $R^{j}$ is

$$
\begin{aligned}
\hat{R}^{j}(t)=\frac{\sigma_{y}^{j}}{\left(\sigma_{x}+\sigma_{y}\right)^{j}} e^{\frac{\left(Y_{(1)}-X_{(1)}\right) j}{\sigma_{y}}} \mathrm{I}\left(Y_{(1)} \leq X_{(1)}\right) \\
\quad+\sum_{i=0}^{j}(-1)^{i}\left(\begin{array}{c}
j \\
i
\end{array}\right)\left(\frac{\sigma_{x}}{\sigma_{x}+\sigma_{y}}\right)^{i} e^{-\frac{\left(Y_{(1)}-X_{(1)}\right) i}{\sigma_{x}}} \mathrm{I}\left(Y_{(1)} \geq X_{(1)}\right) .
\end{aligned}
$$


International Journal of Mathematical, Engineering and Management Sciences

Vol. 5, No. 1, 1-12, 2020

https://doi.org/10.33889/IJMEMS.2020.5.1.001

\section{Interval-System Reliability Estimation}

This section, we discuss the UMVUE and MLE of $k$-out-of- $n$ interval-system reliability based on stress-strength events where $X$ (stress) and $Y$ (strength) are independent two-parameter exponential random variables. A system is able to perform its intended function if its strength is greater than the stress imposed upon it. Reliability of the system, $R=P(Y>X)$, is the probability that the strengths of the unit are greater than the stresses.

The $k$-out-of- $n$ interval-system is a system with a series of $n$ events in a given interval of time which successes (or functions) if and only if at least $k$ out of $n$ independent events success (function).

The reliability of the $k$-out-of- $n$ interval-system is the probability that at least $k$ out of $n$ events in a given interval of time success, and is given by

$$
R_{s}(t)=\sum_{i=k}^{n}\left(\begin{array}{l}
n \\
i
\end{array}\right)(R(t))^{i}(1-R(t))^{n-i}
$$

The reliability function of the $k$-out-of- $n$ interval-system (as from Eq. 11) can be rewritten as (Pham, 2010; Pham and Pham, 2010):

$$
R_{S}(t)=\sum_{j=k}^{n}(-1)^{j-k}\left(\begin{array}{c}
j-1 \\
k-1
\end{array}\right)\left(\begin{array}{c}
n \\
j
\end{array}\right) R^{j}(t)
$$

where $R(t)=P(Y>X)$.

\section{UMVUE of $R_{s}(t)$}

Let

$$
\tilde{R}_{S}(t)=\sum_{j=k}^{n}(-1)^{j-k}\left(\begin{array}{c}
j-1 \\
k-1
\end{array}\right)\left(\begin{array}{l}
n \\
j
\end{array}\right) \tilde{R}^{j}(t)
$$

where (from Eq. (7))

$$
\tilde{R}^{j}(t)=\left\{\begin{array}{l}
\frac{(a-j)\left(j \sigma_{x}+m \sigma_{y}\right) \sigma_{y}^{j-1}}{m a\left(\sigma_{x}+\sigma_{y}\right)^{k}} e^{\frac{\left(Y_{(1)}-X_{(1)}\right) j}{\sigma_{y}}} \mathrm{I}\left(Y_{(1)}<X_{(1)}\right) \\
+\sum_{i=0}^{j}(-1)^{i}\left(\begin{array}{c}
j \\
i
\end{array}\right) \frac{(m-i)\left(a \sigma_{x}+i \sigma_{y}\right) \sigma_{x}^{i-1}}{m a\left(\sigma_{x}+\sigma_{y}\right)^{i}} e^{-\frac{i\left(Y_{(1)}-X_{(1)}\right)}{\sigma_{x}}} \mathrm{I}\left(Y_{(1)} \geq X_{(1)}\right) .
\end{array}\right.
$$

Theorem 2: The statistic $\tilde{R}_{s}(t)$ is the UMVUE of system reliability $R_{S}(t)$ where $\sigma_{x}$ and $\sigma_{y}$ are known and $k<\min \{m, a\}$.

Proof: Based on the polynomial combination of UMVUE functions, we can show that the UMVUE of reliability function of $\mathrm{k}$ out of $\mathrm{n}$ interval-system, $R_{s}(t)$, is 
International Journal of Mathematical, Engineering and Management Sciences

Vol. 5, No. 1, 1-12, 2020

https://doi.org/10.33889/IJMEMS.2020.5.1.001

$$
\tilde{R}_{S}(t)=\sum_{j=k}^{n}(-1)^{j-k}\left(\begin{array}{c}
j-1 \\
k-1
\end{array}\right)\left(\begin{array}{c}
n \\
j
\end{array}\right) \tilde{R}^{j}(t)
$$

where $\tilde{R}^{j}(t)$ is given in Eq. (7).

Q.E.D.

\section{MLE of $R_{s}(t)$}

Hence, the MLE of the system reliability $R_{S}(t)$, where $\sigma_{x}$ and $\sigma_{y}$ are known, is given by

$$
\hat{R}_{S}(t)=\sum_{j=k}^{n}(-1)^{j-k}\left(\begin{array}{c}
j-1 \\
k-1
\end{array}\right)\left(\begin{array}{l}
n \\
j
\end{array}\right) \hat{R}^{j}(t)
$$

where (also from Eq. (10))

$$
\hat{R}^{j}(t)=\frac{\sigma_{y}^{j}}{\left(\sigma_{x}+\sigma_{y}\right)^{j}} e^{\frac{\left(Y_{(1)}-X_{(1)}\right) j}{\sigma_{y}}} \mathrm{I}\left(Y_{(1)}<X_{(1)}\right)+\sum_{i=0}^{j}(-1)^{i}\left(\begin{array}{l}
j \\
i
\end{array}\right)\left(\frac{\sigma_{x}}{\sigma_{x}+\sigma_{y}}\right)^{i} e^{-\frac{\left(Y_{(1)}-X_{(1)}\right) i}{\sigma_{x}}} \mathrm{I}\left(Y_{(1)} \geq X_{(1)}\right) .
$$

\section{Applications}

One in every four deaths in the United States occurs as a result of heart disease (CDC NCHS, 2015). Monitoring your heart rate can help prevent heart complications. The heart rate is one of the important indicators of health in the human body (Perry et al., 2019). It measures the number of times the heart beats per minute. Stress tests are carried out to measure the heart's ability to respond to external/emotion stress in a controlled environment. The stresses induced by exercise on a treadmill monitoring the average heart rate in 12-15 second periods were found as follows:

$15.1,12.3,19.8,13.0,12.5,13.3,18.6,17.2,18.7,22.4,14.6$, and 15.1 (per 15-second period).

The values of normal heart rate of an individual on tests found by monitoring 15-15 second periods (multiply by four to obtain BPM) were:

$19.2,24.2,21.1,16.7,17.6,14.3,22.4,20.8,14.6,17.7,22.7,15.9,19.2,15.3$, and 25.2 (per 15second period)

Given $m=12, a=15, k=2, n=3, \sigma_{x}=3.5$, and $\sigma_{y}=3.7$. Here $X_{(1)}=12.3, Y_{(1)}=14.3$. We now calculate the reliability of 2-out-of-3 interval-system in a given interval of 45 seconds.

Calculate UMVUE

Since $Y_{(1)}=14.30>X_{(1)}=12.30$, from Eq. (14), 
International Journal of Mathematical, Engineering and Management Sciences

Vol. 5, No. 1, 1-12, 2020

https://doi.org/10.33889/IJMEMS.2020.5.1.001

$$
\begin{aligned}
\tilde{R}^{j}(t) & =\sum_{i=0}^{j}(-1)^{i}\left(\begin{array}{c}
j \\
i
\end{array}\right) \frac{(m-i)\left(a \sigma_{x}+i \sigma_{y}\right) \sigma_{x}^{i-1}}{m a\left(\sigma_{x}+\sigma_{y}\right)^{i}} e^{-\frac{i\left(Y_{(1)}-X_{(1)}\right)}{\sigma_{x}}} \\
& =\sum_{i=0}^{j}(-1)^{i}\left(\begin{array}{c}
j \\
i
\end{array}\right) \frac{(12-i)(15(3.5)+i(3.7))(3.5)^{i-1}}{12(15)(3.5+3.7)^{i}} e^{-\frac{i(14.3-12.3)}{3.5}} \\
& =\sum_{i=0}^{j}(-1)^{i}\left(\begin{array}{c}
j \\
i
\end{array}\right) \frac{(12-i)(52.5+3.7 i)(3.5)^{i-1}}{180(7.2)^{i}} e^{-0.5714 i} .
\end{aligned}
$$

So, we obtain

$$
\tilde{R}^{j}(t)= \begin{cases}0.5328917 & \text { for } j=2 \\ 0.3880224 & \text { for } j=3\end{cases}
$$

Thus, the reliability of 2-out-of-3 interval-system in a given interval of 45 seconds is

$$
\begin{aligned}
\tilde{R}_{S}(t) & =\sum_{j=2}^{3}(-1)^{j-2}\left(\begin{array}{l}
j-1 \\
2-1
\end{array}\right)\left(\begin{array}{l}
3 \\
j
\end{array}\right) \tilde{R}^{j}(t) \\
& =3 \tilde{R}^{2}(t)-2 \tilde{R}^{3}(t) \\
& =3(0.5328917)-2(0.3880224) \\
& =0.8226303 .
\end{aligned}
$$

Calculate MLE

From Eq. (15), the MLE of 2-out-of-3 interval-system reliability $R_{S}(t)$ is given by

$$
\hat{R}_{S}(t)=\sum_{j=2}^{3}(-1)^{j-2}\left(\begin{array}{l}
j-1 \\
2-1
\end{array}\right)\left(\begin{array}{l}
3 \\
j
\end{array}\right) \hat{R}^{j}(t)=3 \hat{R}^{2}(t)-2 \hat{R}^{3}(t)
$$

Since $Y_{(1)}=14.30>X_{(1)}=12.30$ and from Eq. (10), we obtain

$$
\begin{aligned}
\hat{R}^{j}(t) & =\sum_{i=0}^{j}(-1)^{i}\left(\begin{array}{l}
j \\
i
\end{array}\right)\left(\frac{3.5}{3.5+3.7}\right)^{i} e^{-\frac{(14.3-12.3) i}{3.5}} \\
& =\sum_{i=0}^{j}(-1)^{i}\left(\begin{array}{l}
j \\
i
\end{array}\right)(0.486111)^{i} e^{-0.5714286 i}
\end{aligned}
$$

then

$$
\hat{R}^{j}(t)= \begin{cases}0.5263275 & \text { for } \mathrm{j}=2 \\ 0.3818423 & \text { for } \mathrm{j}=3 .\end{cases}
$$


International Journal of Mathematical, Engineering and Management Sciences

Vol. 5, No. 1, 1-12, 2020

https://doi.org/10.33889/IJMEMS.2020.5.1.001

Hence,

$$
\begin{aligned}
\hat{R}_{S}(t) & =3 \hat{R}^{2}(t)-2 \hat{R}^{3}(t) \\
& =3(0.5263275)-2(0.3818423) \\
& =0.8152979 .
\end{aligned}
$$

Thus, the UMVUE and MLE reliability of 2-out-of-3 interval system $R_{S}(t)$ are 0.8226303 and 0.8152979 , respectively. From the data on the stress and strength tests measuring the heart rate conditions, the reliability estimates of stress-strength 2-out-of-3 interval-system based on the UMVUE and MLE are $\tilde{R}_{S}(t)=0.8226303$ and $\hat{R}_{S}(t)=0.8152979$, respectively. As expected, there is difference between $\hat{R}_{S}(t)$. and $\hat{R}_{S}(t)$. Comparing $\hat{R}_{S}(t)$. and $\hat{R}_{S}(t)$. We note that $\hat{R}_{S}(t)$. is unbiased while $\hat{R}_{S}(t)$. is not. A general approach of comparison between an unbiased estimator and a biased estimator is to compute the mean squared error (MSE) of the two estimators $\hat{R}_{S}(t)$. and $\hat{R}_{S}(t)$. It can be shown that the UMVUE of $R_{S}(t)$ has smaller MSE than the MLE of $R_{S}(t)$. Based on this data set, the UMVUE of $R_{S}(t)$ is slightly larger than the MLE of $R_{S}(t)$. It is worth to note that, in general, the UMVUE of $R_{S}(t)$ is not necessary to be larger than the MLE of $R_{S}(t)$.

\section{Conclusion}

In this paper we discussed the UMVUE and MLE reliability estimates of $k$-out-of- $n$ intervalsystem based on stress-strength inference events where the stress random variable $X$ and strength random variable $Y$ are independent with two-parameter exponential distributions. An application in human heart conditions is also discussed. In this paper, we assumed that all the events in a given interval of time are statistically independent. It would be very interesting to study as a future research problem when the events in a given interval of time are not independent and both $\sigma_{x}$ and $\sigma_{y}$ are unknown.

\section{Conflict of Interest}

The author confirms that there is no conflict of interest to declare for this publication.

\section{Acknowledgements}

The author would like to appreciate the effort from editors and reviewers. This research did not receive any specific grant from funding agencies in the public, commercial, or not-for-profit sectors.

\section{References}

Aminzadeh, M.S. (1997). Estimation of reliability for exponential stress-strength models with explanatory variables. Applied Mathematics and Computation, 84(2-3), 269-274.

Beg, M.A. (1980). On the estimation of $\operatorname{Pr}\{\mathrm{Y}<\mathrm{X}\}$ for the two-parameter exponential distribution. Metrika, 27(1), 29-34.

Bhattacharyya, G.K., \& Johnson, R.A. (1974). Estimation of a reliability in a multi-component stressstrength model. Journal of the American Statistical Association, 69(348), 966-970. 
International Journal of Mathematical, Engineering and Management Sciences

Vol. 5, No. 1, 1-12, 2020

https://doi.org/10.33889/IJMEMS.2020.5.1.001

CDC, NCHS (2015), Underlying Cause of Death 1999-2013 on CDC WONDER Online Database, released 2015. Data are from the Multiple Cause of Death Files, 1999-2013, as compiled from data provided by the 57 vital statistics jurisdictions through the Vital Statistics Cooperative Program. Accessed Feb. 3, 2015.

Chao, A. (1982). On comparing estimators of $\operatorname{Pr}\{\mathrm{Y}<\mathrm{X}\}$ in the exponential case. IEEE Transactions on Reliability, 31(4), 389-392.

Guo, H., \& Krishnamoorthy, K. (2004). New approximate inferential methods for the reliability parameter in a stress-strength model: the normal case. Communications in Statistics-Theory and Methods, 33(7), 1715-1731.

Hanagal, D.D. (2003) Estimation of system reliability in multi-component stress strength models. Journal of Indian Statistical Association, 41(1-7), 24.

Kotz, S., Lumelskii, Y., \& Pensky, M. (2003). The stress-strength model and its generalizations: theory and applications. World Scientific, Singapore.

Kunchur, S.H., \& Munoli, S.B. (1993). Estimation of reliability for a multi-component survival stressstrength model based on exponential distributions. Communications in Statistics-Theory and Methods, 22(3), 769-779.

Kundu, D., \& Gupta, R.D. (2005). Estimation of $\mathrm{P}[\mathrm{Y}<\mathrm{X}]$ for generalized exponential distribution. Metrika, 61(3), 291-308.

Laurent, A.G. (1963). Conditional distribution of order statistics and distribution of the reduced $i^{\text {th }}$ order statistic of the exponential model. The Annals of Mathematical Statistics, 34(2), 652-657.

Lehmann, E.L., \& Casella, G. (1998). Theory of point estimation. 2nd ed., Springer, New York, NY.

Perry, S., Khovanova, N.A., \& Khovanov, I.A. (2019). Control of heart rate through guided high-rate breathing. Scientific Reports, 9(1), 1545. DOI: 10.1038/s41598-018-38058-5.

Pham, H. (2010). On the estimation of reliability of k-out-of-n systems. International Journal of Systems Assurance Engineering and Management, 1(1), 32-35.

Pham, H. (2018, August). Stress-strength interval-system reliability model and its applications in heart conditions, In $24^{\text {th }}$ ISSAT International Conference on Reliability and Quality in Design (pp. 260-263). International Society of Science and Applied Technologies. Toronto, Canada.

Pham, H., \& Pham, H.Jr. (2010). Improving energy and power efficiency using NComputing and approaches for predicting reliability of complex computing systems. International Journal of Automation and Computing, 7(2), 153-159.

Rutemiller, H.C. (1966). Point estimation of reliability of a system comprised of k elements from the same exponential distribution. Journal of the American Statistical Association, 61(316), 1029-1032.

Saraçoglu, B., \& Kaya, M.F. (2007). Maximum likelihood estimation and confidence intervals of system reliability for Gompertz distribution in stress-strength models. Selçuk Journal of Applied Mathematics, $8(2), 25-36$.

Weerahandi, S., \& Johnson, R.A. (1992). Testing reliability in a stress-strength model when $X$ and $Y$ are normally distributed. Technometrics, 34(1), 83-91.

Wei, J., Pan, Z., Lin, X., Qin, D., Zhang, A., \& Shi, L. (2019). Copula-function-based analysis model and dynamic reliability of a gear transmission system considering failure correlations, Fatigue \& Fracture of Engineering Materials \& Structures, 42(1), 114-128.

You, D., \& Pham, H. (2016). Reliability analysis of the CNC system based on field failure data in operating environments. Quality and Reliability Engineering International, 32(5), 1955-1963. 
International Journal of Mathematical, Engineering and Management Sciences

Vol. 5, No. 1, 1-12, 2020

https://doi.org/10.33889/IJMEMS.2020.5.1.001

\section{APPENDIX}

Proof of Theorem 1: We need to show that

$$
E\left(T_{k}\right)=R^{k}
$$

where $R^{k}=[\mathrm{P}(\mathrm{Y}>\mathrm{X})]^{k}$ as given in Eq. (6).

When $\mu_{y}>\mu_{x}$ : We have

$$
\begin{aligned}
E\left(T_{k}\right)= & \frac{(a-k)\left(k \sigma_{x}+m \sigma_{y}\right) \sigma_{y}^{k-1}}{m a\left(\sigma_{x}+\sigma_{y}\right)^{k}}\left(\int_{-\infty}^{\mu_{y}-\mu_{x}} \frac{m a}{a \sigma_{x}+m \sigma_{y}} e^{-\frac{m\left(\mu_{y}-\mu_{x}\right)}{\sigma_{x}}+w\left(\frac{k}{\sigma_{y}}+\frac{m}{\sigma_{x}}\right)} d w\right) \\
& +\left(\sum_{i=0}^{k}(-1)^{i}\left(\begin{array}{c}
k \\
i
\end{array}\right) \frac{(m-i)\left(a \sigma_{x}+i \sigma_{y}\right) \sigma_{x}^{i-1}}{m a\left(\sigma_{x}+\sigma_{y}\right)^{i}}\right) \\
& \left(\int_{0}^{\mu_{y}-\mu_{x}}\left(\frac{m a}{a \sigma_{x}+m \sigma_{y}}\right) e^{-\frac{m\left(\mu_{y}-\mu_{x}\right)}{\sigma_{x}}+\frac{(m-i) w}{\sigma_{x}}} d w+\left(\frac{m a}{a \sigma_{x}+m \sigma_{y}}\right) \int_{\left(\mu_{y}-\mu_{x}\right)}^{\infty} e^{\frac{a\left(\mu_{y}-\mu_{x}\right)}{\sigma_{y}}-\left(\frac{a}{\sigma_{y}}+\frac{i}{\sigma_{x}}\right) w} d w\right) .
\end{aligned}
$$

By taking the integrals and some algebra simplifications, we obtain

$$
E\left(T_{k}\right)=[P(Y>X)]^{k}+\frac{B \sigma_{x}}{\left(a \sigma_{x}+m \sigma_{y}\right)\left(\sigma_{x}+\sigma_{y}\right)^{k}} e^{-\frac{m\left(\mu_{y}-\mu_{x}\right)}{\sigma_{x}}}
$$

where

$$
B=(a-k) \sigma_{y}^{k}-\sum_{i=0}^{k}(-1)^{i}\left(\begin{array}{l}
k \\
i
\end{array}\right)\left(a \sigma_{x}+i \sigma_{y}\right)\left(\sigma_{x}+\sigma_{y}\right)^{k-i} \sigma_{x}^{i-1}
$$

After some simplifications, we can show that $B=0$. Thus,

$$
E\left(T_{k}\right)=[P(Y>X)]^{k} \quad \text { for } \mu_{y}>\mu_{x} .
$$

When $\mu_{y} \leq \mu_{x}$ : We have

$$
\begin{aligned}
E\left(T_{k}\right)= & \frac{(a-k)\left(k \sigma_{x}+m \sigma_{y}\right) \sigma_{y}^{k-1}}{m a\left(\sigma_{x}+\sigma_{y}\right)^{k}} \\
& \left\{\int_{-\infty}^{\mu_{y}-\mu_{x}} \frac{m a}{a \sigma_{x}+m \sigma_{y}} e^{-\frac{m\left(\mu_{y}-\mu_{x}\right)}{\sigma_{x}}+w\left(\frac{k}{\sigma_{y}}+\frac{m}{\sigma_{x}}\right)} d w+\int_{\mu_{y}-\mu_{x}}^{0} \frac{m a}{a \sigma_{x}+m \sigma_{y}} e^{\frac{a\left(\mu_{y}-\mu_{x}\right)}{\sigma_{y}}-w\left(\frac{a}{\sigma_{y}}-\frac{k}{\sigma_{y}}\right)} d w\right\} \\
+ & \left(\sum_{i=0}^{k}(-1)^{i}\left(\begin{array}{l}
k \\
i
\end{array}\right)\left(\frac{(m-i)\left(a \sigma_{x}+i \sigma_{y}\right) \sigma_{x}^{i-1}}{m a\left(\sigma_{x}+\sigma_{y}\right)^{i}}\right)\left(\int_{0}^{\infty} \frac{m a}{a \sigma_{x}+m \sigma_{y}} e^{\frac{a\left(\mu_{y}-\mu_{x}\right)}{\sigma_{y}}-w\left(\frac{a}{\sigma_{y}}+\frac{i}{\sigma_{x}}\right)} d w\right) .\right.
\end{aligned}
$$


International Journal of Mathematical, Engineering and Management Sciences

Vol. 5, No. 1, 1-12, 2020

https://doi.org/10.33889/IJMEMS.2020.5.1.001

After taking integrals and some simplifications, we obtain

$$
E\left(T_{k}\right)=[P(Y>X)]^{k}+\frac{A \sigma_{y}}{a \sigma_{x}+m \sigma_{y}} e^{\frac{a\left(\mu_{y}-\mu_{x}\right)}{\sigma_{y}}}
$$

where

$$
A=\frac{1}{\left(\sigma_{x}+\sigma_{y}\right)^{k}}\left(\sum_{i=0}^{k}(-1)^{i}\left(\begin{array}{l}
k \\
i
\end{array}\right)(m-i) \sigma_{x}^{i}\left(\sigma_{x}+\sigma_{y}\right)^{k-i}-\sigma_{y}^{k-1}\left(k \sigma_{x}+m \sigma_{y}\right)\right) .
$$

It can be shown that,

$$
A=\frac{1}{\left(\sigma_{x}+\sigma_{y}\right)^{k}}\left(\sum_{i=0}^{k}(-1)^{i}\left(\begin{array}{l}
k \\
i
\end{array}\right)(m-i) \sigma_{x}^{i}\left(\sigma_{x}+\sigma_{y}\right)^{k-i}-\sigma_{y}^{k-1}\left(k \sigma_{x}+m \sigma_{y}\right)\right)=0
$$

Therefore,

$$
E\left(T_{k}\right)=[P(Y>X)]^{k} \text { for } \mu_{y} \leq \mu_{x}
$$

Hence, $T_{k}$ is an unbiased estimator of $R^{k}$, and from the Lehmann-Scheffe theorem (Lehmann and Casella, 1998), $T_{k}$ is the UMVUE of $R^{k}$ where

Q.E.D.

$$
R^{k}=[P(Y>X)]^{k} \text {. }
$$

